\title{
The grand challenge for Frontiers in Emotion Science
}

\author{
Beatrice de Gelder ${ }^{1,2 *}$ \\ 1 Martinos Centre for Biomedical Imaging, Massachusetts General Hospital and Harvard Medical School, Charlestown, MA, USA \\ 2 Cognitive and Affective Neuroscience Laboratory, Tilburg University, Tilburg, Netherlands \\ *Correspondence: degelder@uvt.nl
}

Everyday explanations of behavior that one way or another appeal to emotions are ubiquitous and there appear almost always to be three components to them. First, there is a reference to some significant event in the environment or in the person (a source of danger in the environment or the sight of an angry person). The second component refers to the effects in the mind, brain, and body of the person to whom we attribute an emotion or who attributes an emotion to himself. Typical examples of a change of mind are a sudden state of fear or disgust, or physiological changes like in breathing rhythm, feelings of excitement and changes in behavior like running and a number of actions. The third component consists in reference to the goal of the events. This involves typically a statement of what is good or bad for the organism, how the events that trigger emotions appear to contribute significantly, positively, or negatively, to somebody's overall life goals. These three components, separately or in various combinations, are the pillars of our everyday language of emotions. Is the science of emotion wedded to this language and the worldview it embodies? If not, it may be easier to develop new scientific emotion theories, but the price to pay is a disconnection from subjective experience. Emotion science may be in a unique position in that subjective experience is part and parcel of its subject matter.

For many decades mainstream science has been prejudiced against the possibility of a serious, respectable scientific study of emotions. Much as we all pay lip service to an evolutionary perspective on mind, brain, and behavior, many of us still somehow exclude emotions from this evolutionary objectivism. Emotions seem somehow too dense with daily psychological reality to promise solid scientific insights. To some extent this situation is related to the vagueness of the core notions used in affective science. For example, it is often unclear what the difference is between terms like emotions, feelings, affect, subjective experience, and other related ones (Russell and
Feldman Barrett, 1999). Researchers outside the field of emotion sometimes blame the confusion surrounding the concept of emotion for this state of affairs. The concept of emotion is ill defined and there is the feeling both inside and outside the community that the first issue on the agenda should be to craft a definition of what emotions are (Kleinginna and Kleinginna, 1981). But this is most probably an illusion. One hardly needs to glance at the situation in other disciplines to see that notions like nature, environment etc., are equally open-ended and that this does not hinder scientific research. The history of science shows that definitions are the result rather that the beginning of scientific theories.

From the very beginning of scientific psychology with Wundt (1897) and of emotion theory with Darwin (1872) and James (1890) two different ways of thinking about emotions have been around. Wundt paid attention to the phenomenal experience using quantitative and qualitative descriptors that hold across all emotions. Darwin instead concentrated on the discrete muscle patterns associated with some of the major emotions, while James focused on the causal relation between bodily changes and emotional feelings. So, from the very beginning emotion theories were addressing two very different sides of the same coin. At present, the gap between movement patterns and phenomenal experience remains as theoretically important and as hard to bridge as it was a hundred years ago. As a matter of fact, with the advent of an increasingly broad array of new measuring tools the gap has opened wider and wider. More layers than ever imagined now become visible but the task of relating the different strata to each other has become more daunting than ever.

We would like to mark the launch of Frontiers in Emotion Science by looking forward and formulate a few landmark questions including some that have not attracted much attention so far: How many emotions are there, are all emotions alike, what is there to measure, how do we measure emotions, what does interdisciplinarity mean, how important is interaction, what are the issues for modeling emotions, what is the part of context, do feelings matter, consciousness, and emotions.

\section{HOW MANY EMOTIONS ARE THERE? HOW DO WE COUNT EMOTIONS?}

A question that intrigues the layman and plagues scientists is how many emotions there really are. Are fears and panic the same emotion, different only in intensity? Social anxiety hinge on the same basis as spider phobia, only differing in object? Are embarrassment and guilt the same, again only different in intensity? Is empathy an emotion? As soon as we begin to answer such questions we get boggled down in linguistic and terminological nightmares. Older schools of philosophical psychologists as we find them, e.g., in Germany in the eighteenth and nineteenth century, have provided elaborate classifications. More empirically minded authors have from the beginning been torn between so called dimensional accounts, organizing the variety of emotional states along different continua or in discrete categories possibly structured as the basic vs. the extended emotion system (Davidson, 1995). A dimensional account that is seemingly the best supported in recent reach consists of two dimensions, "arousal" (or intensity) and "valence" (positive or negative, pleasing or unpleasant (Plutchik, 1980). Other authors used different labels for the two dimensions and contrast approach and withdrawal (Davidson, 1995) Simple dimensional contrasts work reasonably well for pairing with behavioral measures with emotion labels and with physiological measures. However, a common critique is that it just will not work to map the variety of behaviors, experiences and feelings typically associated with different emotions, along such simple dimensions. The most famous spokesperson of a discrete counting method for emotions is Ekman (1999). His view is associated with that of six basic emotions, which are happiness, surprise, fear, anger, 
disgust, and sadness. As noted above, his view is that since emotions are evolutionary shaped biological properties of the organism, they have to be universal within the same species. Therefore his motivation was to search for such universal emotions and his method was to look for facial muscle patterns. An underlying assumption is that fixed muscle patterns reflect pre-wired mental states with characteristic expressions that are in turn well characterized by the emotion words we use for them. Another example is the theory developed by Panksepp (1998). Similar to the position taken by LeDoux (1996), Panksepp believes that there are a few clear and separate emotions and that the best chance for scientific progress is to tackle each separately. LeDoux argues that fear is in this respect the most promising candidate. Panksepp has argued for four basic emotional systems (fear, seeking, panic, and rage). These are selected on the basis of their purported neurobiological profile (including characteristic behavior as well as neurobiological signatures). The point to note is that each of these positions is welded to a specific research agenda that has proven successful in the past, for addressing the questions of the day and using the methods of the times. But science progresses by discovering new questions rather than only by answering existing ones.

\section{WHAT TO MEASURE?}

We just discussed the near impossibility of quantifying emotions outside any theoretical context. A different issue to bring up before we look into emotion measurement issues properly speaking is what to measure. By and far, most emotion theories aim at measuring emotions and in the process just concentrate on one kind of emotional signal, mostly the face. In the large majority of the studies the face is used as a stimulus to trigger an emotional state in the observer (de Gelder et al., 2006). The focus of measuring emotions is then the experience triggered by seeing the emotional stimulus. That experience is seen as encompassing different layers including changes in physiological state, states of action readiness, and subjective experience. Depending on the theory, it also includes evaluation of appraisal of the emotion triggering event and its initial effects on the organism (Scherer, 2009). But with the exception of Paul Ekman's work (and to some extent Dimberg's (1982) the facial behavior itself is not measured.
Modern emotion researchers have not yet clarified the link between emotions and a basic repertoire of actions like approach, avoidance, search, or fear. For example, the question as to whether specific body movements are associated with distinct emotional states has rarely been addressed (de Gelder, 2006; de Gelder et al., 2006). This is surprising given that Aristotle, in his treatise on Physiognomics, was already convinced that the body as a whole expressed emotional states and that facial expressions were simply a part of that overt behavior. Since the early work of Hess (Hess and Brügger, 1943) who observed that electrical stimulation of the tectum and related structures induced characteristic postures and even behaviors expressing emotions Mori in Japan has conducted a systematic analysis of the neural basis of some aspects of emotional expressions of the whole body in the cat (Mori et al., 1999). The important contribution of this series of studies is to suggest that bodily expressions are produced by an output of the emotional system to brain stem and mesencephalic centers triggering behaviors that belong to motor repertoire of the animal. A grand exception is the approach by Frijda (2007). The central concept of all his theoretical work on emotions is that emotions are states of action readiness.

\section{MULTIDISCIPLINARITY OR INTERDISCIPLINARITY?}

The general perception is that the most urgent task is that of measuring the mind at the different levels and with all the different methodologies currently available and to integrate the results across the different methods (behavioral, personality questionnaires, physiology, neurofunctional correlates, genetics, neuropsychology). In the last decade emotion research has concentrated on brain activity generated by the perception of still images of facial expressions and this has already produced models of the brain network involved in perceiving emotional signals typically provide by facial expressions (Haxby et al., 2000; Adolphs, 2002; de Gelder et al., 2003) and outlined the role of several structures such as the amygdala, orbito-frontal, or cingulate cortex. Recent studies broaden the perspective by drawing attention to extra-visual areas like insula (Craig, 2009), sensory-motor cortex (Adolphs et al., 2000; Sato et al., 2004).
A more evolutionarily plausible approach to emotions puts the adaptive dimension of emotions on the forefront and emphasizes the close relationship between emotion and action (Panksepp, 1998; de Gelder, 2006; Frijda, 2007; Tamietto and de Gelder, 2010). The study of affective processes is by necessity interdisciplinary and this is central goal of the burgeoning field of affective neurosciences. At present, agreeing on an a priori definition of emotion than by drafting an innovative and theoretically wellfounded research agenda bringing affective neuroscience in line with the biological sciences will no so much provide this. A major tasks is to unlock the information contained at the different levels at which emotional behavior can currently be measured such that we begin to understand how the different levels of affective existence. Measuring affective processing then takes the form of building bridges between the levels of processing (personal, sub personal, behavioral, physiological, etc) and the measures (psychophysics, lesion studies, neurodegenerative disorders, brainimaging, etc).

\section{MENTALISM OR BIOLOGISM?}

Clarifying the focus of affective science by (re)starting from an evolutionary perspective rather than a Cartesian one. A sophisticated analysis of emotional behavior must allow us to overcome the misleading distinction or even the absolute gap between inner mental life and outer behavioral and physiological manifestations that we measure but whose pertinence for the real subjective emotional experience we tend to doubt.

\section{MULTISENSORY CHARACTER OF AFFECTIVE SIGNALING SYSTEMS}

In human research tradition has favored the visual system over the auditory, the auditory over the haptic, the haptic over the olfactory. There is at present very little research onto multisensory emotion signals, although real life experience certainly convinces us of the importance of taste and smell. For example, the amygdala plays a critical role not just in processing visual information like fearful facial or bodily expressions but equally auditory information like voice prosody. This may be viewed as an indication that the message is more important than the medium and that the functional organization of the brain is at least as sensitive to the message than to the medium. 


\section{THE MEDIUM AND THE MESSAGE OF AFFECTIVE SIGNALS}

Human emotion investigations have concentrated on one medium of affective communication, the face. Our current theories and insights may reflect this bias and may block the view on the emotion message. Other channels of communication like the whole body and the voice need to be part of the scope of emotion theories and importantly, of the envisaged applications including clinical ones. We do not know at present whether deficits or biases in one channel also exist in others. For example, emotion perception deficits in autism or in schizophrenia are reported for facial expressions, but less so for bodily expressions, at least in explicit behavioral recognition tasks.

\section{PHYLOGENETIC BRAIN SPECIALIZATION AND LATERALITY, BRAIN ARCHEOLOGY, AND RECYCLING}

Cross-species studies are much needed as current models of human emotion processing typically are a mix of insights from animal research (e.g., about brain areas and their connectivity), physiological, and behavioral studies for which there is currently very little evidence in humans. One obstacle is the tendency to conflate matters of form and matters of function and this leads to overgeneralizations without much empirical support. Cross species studies are difficult though. Human emotion labels do not readily apply to animal observations. The obstacles are not just terminological. Simple words like angry, fear of happy are easy to use when human observers classify human vocalizations. When we use them, we are aware of anthropomorphizing as a means what we see and feel to be the case in the animal. But still the untrained observer is at loss to apply these words to monkey vocalizations. It does not help much to look for similarities in facial muscle patterns. Fear, joy, and anger facial expressions are quite different in human or animal displays and these are only just the most obvious emotion labels.

\section{REALISM, NATURALISM, AND CULTURALISM}

Different emotional signing systems (face, body, voice) need to be investigated in relation to their naturalistic context as they have intrinsic links with each other, in per- ception as well as in production. Context, phylogenetically as well as ontogenetically and context of acquisition of affective signal ability are important and so is the context of reading the emotional signals. Neither has received much attention so far by researchers.

Computational modeling of emotional processes must reckon with the likelihood that the affective mind is distributed over different relatively autonomous subsystems (different sensory systems, different carriers of affective information within the same sensory system, different types of processing, e.g., explicit or implicit within and across sensory systems; different context of reading affective signals) that are only partly coherent and probably obey principles of local optimization of information determined by a host of context factors.

\section{INTERACTION MAKES OR BRAKES EMOTIONAL COMMUNICATION}

The long prevailing mentalism loosely associated with Descartes (but part of any approach of emotions that is build on the distinction between emotion and cognition), viewed emotions as individual subjective mental states. More biologically oriented analysis will need to reveal the interactive nature of expressions. For example, in neuropsychology an interesting attempt was made by Ajurriaguera to study the postures of tenderness between a mother and her child. The originality of his approach was to consider body expression of emotion as an interactive link between two people. This remained an isolated theme that is a promising to be explored in contemporary research.

\section{EMOTIONS AND CONSCIOUSNESS}

The relation between emotion and consciousness is a complicated one. It has long been argued that the term non-conscious emotions is an oxymoron, the argument being that emotions require subjective experience hence by definition require awareness. For some theorists emotional experience follows emotional reflex actions while for others it is part of decision-making and precedes action. A classical position on this is the James-Lange theory postulating that our emotional feelings and experience follow from awareness of our automatic reaction to affective signals.
In recent years, these models have been rediscovered and extended by neuroscientists, notably Damasio and colleagues in their formulation of the somatic marker hypothesis (Damasio, 1994). This idea is also present in contemporary perspectives on consciousness, including Edelman's theory of neural Darwinism (Tononi and Edelman, 1998). New findings on nonconscious affective perception challenge this view and to us indicate that we cannot treat consciousness as a unitary phenomenon (Tamietto and de Gelder, 2010). A distinction is needed between affective consciousness and cognitive consciousness. Cognitive consciousness is sometimes used interchangeably with attention and both presume partial or complete visual awareness of the stimulus. The concept of affective consciousness has a long but different history. In part this was due to the fact that affective awareness was in the past systematically relegated to dubious phenomena like gut feelings, hunched, or wordless intuitions. Empirical evidence concerning the dependence between somatic responses and affective experience is at present still ambiguous. Do we have immediate explicit access to autonomic and somatic activity as suggested by William James? It is unclear whether internal cues that produce feelings of arousal are perceived without being attended to and if so, whether such perception is accurate. Bodily sensations are rarely experienced consciously or with perfect fidelity (Barrett et al., 2007), yet they are nonetheless continually detected and internally represented and they are the basis for subjective experience of affect in humans. There is a substantial literature on consciousness including by emotion researchers yet a lot of it seems to concern cognitive awareness of emotions. We may need to explore a different approach that focuses on affective awareness and feelings and different epistemological criteria about subjective certainty may then apply here.

In conclusion, pushed forward by the newly perceived importance of the field and by many novel questions confronting it, Frontiers in Emotion Science will broaden its scope in the coming decades. Rather that figuring as one of the subfields of psychological science, the issues that Frontiers in Emotion Science deals with may increasingly fuel scientific progress in a host of other fields. 


\section{REFERENCES}

Adolphs, R. (2002). Neural systems for recognizing emotion. Curr. Opin. Neurobiol. 12, 169-177.

Adolphs, R., Damasio, H., Tranel, D., Cooper, G., and Damasio, A. R. (2000). A role for somatosensory cortices in the visual recognition of emotion as revealed by three-dimensional lesion mapping. J. Neurosci. 20 , 2683-2690.

Barrett, L. F., Mesquita, B., Ochsner, K. N., and Gross, J. J. (2007). The experience of emotion. Annu. Rev. Psychol. 58, 373-403.

Craig, A. D. (2009). How do you feel-now? The anterior insula and human awareness. Nat. Rev. Neurosci. 10, 59-70.

Damasio, A. R. (1994). Descartes' Error: Emotion, Reason, and The Human Brain. New York: G.P. Putnam's Sons.

Darwin, C. (1872). The Expression of Emotions in Man and Animals. Oxford, UK: Oxford University Press.

Davidson, R. J. (1995). "Cerebral asymmetry, emotion, and affective style," in Brain Asymmetry, eds R. J. Davidson and K. Hugdahl (Cambridge, MA: MIT Press), 361-388.

de Gelder, B. (2006). Towards the neurobiology of emotional body language. Nat. Rev. Neurosci. 7, 242-249.

de Gelder, B., Frissen, I., Barton, J., and Hadjikhani, N. (2003). A modulatory role for facial expressions in prosopagnosia. Proc. Natl. Acad. Sci. U.S.A. 100, 13105-13110.

de Gelder, B., Meeren, H. K., Righart, R., van den Stock, J., van de Riet, W. A., and Tamietto, M. (2006). Beyond the face: exploring rapid influences of context on face processing. Prog. Brain Res. 155, 37-48.

Dimberg, U. (1982). Facial reactions to facial expressions. Psychophysiology 19, 643-647.

Ekman, P. (1999). "Basic emotions," in Handbook of Cognition and Emotion, eds T. Dalgleish and M. J. Power (New York, NY: John Wiley \& Sons Ltd), 45-60.

Frijda, N. H. (2007). The Laws of Emotion. Mahwah: Lawrence Erlbaum Associates.

Haxby, J. V., Hoffman, E. A., and Gobbini, M. I. (2000). The distributed human neural system for face perception. Trends Cogn. Sci. 4, 223-233.

Hess, W. R., and Brügger, M. (1943). Das subkortikale Zentrum der affectiven Abwerreaktion. Helv. Physiol. Pharmacol. Acta 1, 33-52.

James, W. (1890). Principles of Psychology. New York: Holt.

Kleinginna, R. K., and Kleinginna, M. A. (1981). A categorized list of emotion definitions, with suggestions for a consensual definition. Motiv. Emot. 5, 345-379.

LeDoux, J. E. (1996). The Emotional Brain. New York: Simon \& Shuster.

Mori, S., Matsui, T., Kuze, B., Asanome, M., Nakajima, K., and Matsuyama, K. (1999). Stimulation of a restricted region in the midline cerebellar white matter evokes coordinated quadrupedal locomotion in the decerebrate cat. J. Neurophysiol. 82, 290-300.

Panksepp, J. (1998). Affective Neuroscience. New York, NY: Oxford University Press.

Plutchik, R. (1980). Emotion: A Psychoevolutionary Synthesis. New York, NY: Harper and Row.
Russell, J. A., and Feldman Barrett, L. (1999). Core affect, prototypical emotional episodes, and other things called emotion: dissecting the elephant. J. Pers. Soc. Psychol. 76, 805-819.

Sato, W., Kochiyama, T., Yoshikawa, S., Naito, E., and Matsumura, M. (2004). Enhanced neural activity in response to dynamic facial expressions of emotion: an fMRI study. Cogn. Brain Res. 20, 81-91.

Scherer, K. R. (2009). Emotions are emergent processes: they require a dynamic computational architecture. Philos. Trans. R. Soc. Lond. B. Biol. Sci. 364, 3459-3474.

Tamietto, M., and de Gelder, B. (2010). Neural bases of the non-conscious perception of emotional signals. Nat. Rev. Neurosci. 11, 697-709.

Tononi, G., and Edelman, G. M. (1998). Consciousness and complexity. Science 282, 1846-1551.

Wundt, W. (1897). Outlines of Psychology. Leipzig: Wilhelm Engelmann.

Received: 01 September 2010; accepted: 12 October 2010; published online: 02 November 2010

Citation: de Gelder B (2010). The grand challenge for Frontiers in Emotion Science. Front. Psychology. 1:187. doi: 10.3389/fpsyg.2010.00187.

This article was submitted to Frontiers in Emotion Science, a specialty of Frontiers in Psychology.

Copyright (C) 2010 de Gelder. This is an open-access article subject to an exclusive license agreement between the authors and the Frontiers Research Foundation, which permits unrestricted use, distribution, and reproduction in any medium, provided the original authors and source are credited. 